This is an Accepted Manuscript of an article published by Taylor \& Francis in Drugs: Education, Prevention and Policy on 16/11/16, available online:

http://www.tandfonline.com/10.1080/09687637.2016.1252316 


\title{
Attitudes of intravenous drug users in London towards the provision of drug consumption rooms
}

\author{
Georgia Butler, ${ }^{1,} \mathrm{PhD}$, Dita Chapman ${ }^{1}$, BSc, and Philip Terry ${ }^{1} \mathrm{PhD}$
}

${ }^{1}$ Department of Psychology, Kingston University, Penrhyn Road, Kingston-upon-Thames, United Kingdom

Abbreviated Title: Drug Consumption Rooms

All correspondence should be addressed to Dr Georgia Butler at Department of Psychology, Kingston University, Penrhyn Road, Kingston-upon-Thames, Surrey KT1 2EE, United Kingdom

Email: gk.butler@kingston.ac.uk; Tel: +44(0)2084177002. 
Attitudes of intravenous drug users in London towards the provision of drug consumption rooms

\section{Abstract}

Aims: The study investigated the attitudes of intravenous drug users (IDUs) towards the provision of drug consumption rooms (DCRs) in the UK and their willingness to use DCRs. Methods: Participants were 90 methadone-maintained outpatients recruited from a London clinic. A questionnaire asked about their willingness to use a DCR, their views on various rules commonly-implemented by DCRs, and how they believed DCRs might impact on the drug-taking behaviours of drug users and their peers. Findings: A large majority (89\%) expressed willingness to use a DCR and accepted the need for rules such as no drug sharing (84.3\%), no assistance with injecting (81.8\%), compulsory supervision (76.7\%) and compulsory hand washing (92.1\%). However, the IDUs were split over whether injection in the neck or groin should be disallowed and whether certain categories of IDUs (e.g. juveniles, pregnant women) should be excluded from DCRs. Majorities thought it unlikely that DCRs would encourage users to try risker drug preparations (76.6\%), or encourage non injectors to inject for the first time (74.5\%). Conclusions: In a country where DCRs are not available, the study highlights the willingness of IDUs to use a DCR and accept its rules, even for a sample most of whom were not homeless.

\section{Keywords}

Drug consumption rooms; safe injection facilities; intravenous drug users. 
Attitudes of intravenous drug users in London towards the provision of drug consumption rooms.

\section{Introduction}

Some of the most significant problems associated with drug abuse are a consequence of the use of parenteral routes of administration, in particular the intravenous injection of drugs (EMCDDA, 2012). Intravenous administration is common amongst heroin users, for whom the profile of drug use is often long term and involves periods of abstinence and relapse (McLellan, 2000: Hser et al., 2001). Frequent relapse to injecting drug use also occurs during methadone maintenance treatment (Termorshuizen et al., 2005; Gossop et al., 2003). Injecting drugs creates a host of potential problems for the user (Kerr et al., 2006; Hunt, 2006); it also impacts substantially on the wider community in a number of ways (Kerr et al., 2006; Cusick \& Kimber, 2007). For example, tens of thousands of drug injections occur in public places in England every month (IWG, 2006) and this is not exclusively by homeless users. A report ten years ago (IWG, 2006) indicated that 25\% of those living in their own accommodation had injected in a public place in the previous week. Injecting in public places is associated with drug-dealing and the discarding of needles and other drug paraphernalia; these behaviours inevitably create concern among the affected communities (Cusick \& Kimber, 2006). Importantly, for the user, injecting in public places is also associated with an increased risk of needle-sharing, HCV infection and overdose (Latkin et al., 1994).

In adopting public health policies centered on harm reduction, some countries have introduced drug consumption rooms (DCRs; also known as safe injection facilities (SIFs) or medically supervised injecting centers (MSICs) for injecting drug users (IDUs)). These include eight European Countries, where there are 88 DCRs (Woods et al, 2014), and one in each of Australia and Canada. DCRs operate in cities near to drug markets and are legally- 
sanctioned, supervised places where IDUs can inject street-bought drugs in a clean and safe environment. Sterile injecting equipment and safe disposal facilities are provided and staff are available to deal with overdoses or other emergencies (Hedrich, 2004; Dolan et al., 2000). The IDUs may also receive referrals to other services, such as drug treatment programmes. The primary aims of DCRs are to reduce the harms associated with unsupervised injection, such as overdose and syringe sharing (Kerr et al., 2005), and to improve health outcomes for IDUs such as reduced risk of HIV infection (Pinkerton, 2010). They may also have ancillary benefits to the local community, such as reducing drug- related litter (IWG, 2006; KPMG; 2010; Wood et al., 2004). All DCRs have explicit rules with which the users must comply, such as no sharing of drugs, and no injecting in the neck or groin (Hedrich, 2004). Particular entry criteria may also be adopted, for example regular use of heroin or cocaine, and the exclusion of anyone under 18 years old. Non-injecting drug users who want to conduct their first injection at a DCR are also typically excluded (Hedrich, 2004).

At present there are no such facilities in the UK. A report by An Independent Working Group, commissioned by The Joseph Rowntree Foundation (a UK charity working for social change), included reviewing evidence on DCRs in other countries, commissioning research on DCRs, visiting DCRs, and conducting consultations with drug users. After the 20 month review period, it recommended that DCRs be piloted in the UK (IWG, 2006). However, specific ethical concerns were raised by the IWG, in particular that the provision of DCRs might serve to encourage non-injectors or partners of IDUs to inject for the first time, or they might provide a sense of security that could encourage IDUs to try more risky drug taking practices. The IWG recommended that piloting schemes and evaluation could address these concerns. However, pilot schemes have not been trialled in the UK since the report and neither has any research been undertaken to investigate the ethical issues raised.The UK government rejected the IWG recommendations, primarily due to a 
perceived lack of evaluation of DCRs, legal complications (potential conflict with UN conventions or the Misuse of Drugs Act), concerns about how the public would react, the cost of implementation, and the possibility that DCRs might attract drug dealers to an area (Lloyd \& Hunt, 2007). Many of these are indeed contentious issues, but Lloyd and Hunt (2007) have previously argued that government objections to DCRs in 2002 and 2007 carried little weight. Lloyd (2016; as cited in Broe, 2016) noted that in the UK there is a lack of political will for DCRs.

Nevertheless, there are some evaluation data from a number of countries that have set up DCRs. A ten year evaluation of a DCR in Sydney, Australia, reported a decrease in drug overdose deaths: despite a large number of overdoses occurring in the DCR, there had not been a single death onsite. Reduced sightings of injecting and discarded needles/syringes by local residents and businesses were also reported (KPMG, 2010). A number of evaluations of the DCR provision in Vancouver, Canada, also showed positive outcomes, including cleaner injecting practices, reduced injecting in public places, less unsafe syringe disposal and reduced needle sharing (Wood et al., 2004; Petrar et al., 2007). In addition, the facility attracted higher-risk IDUs including public injectors and users at high risk for HIV and overdose (Wood et al., 2005). A review of the literature on DCRs reported that DCRs were successful in several respects, for example by attracting the most marginalized IDUs, facilitating access to health care, and reducing overdoses; these were achieved without an increase in intravenous drug use or criminal activity (Potier, et al., 2014).

The Sydney MSIC evaluation (MSIC, 2003) highlighted a large number of overdoses at the centre and suggested that these may reflect the adoption of riskier drug taking practices (such as using more heroin). However, a Europe-wide report on DCRs found no evidence that such facilities "increase levels of drug use or encourage riskier patterns of use, nor that they increase morbidity and mortality" (Hedrich, 2004; p79). It also concluded that "there is no 
evidence that consumption rooms encourage increased drug use or initiate new users” (Hedrich, p 84). Further research is needed to examine how drug taking practices change in DCRs, whether they help to initiate non-injectors into injecting as well as issues such as their cost-effectiveness and the likely uptake and acceptability of such facilities in the UK.

Previous studies assessing the likelihood of using a DCR have generally reported willingness to use such a facility. In Sydney, $66 \%$ of IDUs who last injected in a private place and $83 \%$ who last injected in a public place reported being willing to use a DCR (van Beek \& Gilmore, 2000). Similarly, in Montreal, Canada, 76\% of IDUs who injected in public or semi-public places reported willingness to use a DCR if it was established (Green, Hankins, Palmer, Bovin \& Platt, 2004). Fry (2002) found that the majority (89\%) of IDUs interviewed (in Melbourne, Australia) would be willing to use a DCR if it was near to where they purchase and use drugs. Large majorities also reported willingness to use a DCR even if rules were implemented, such as hand washing prior to injecting, no assisting of others to inject, and supervision by staff. In Vancouver, 92\% of the sample reported being willing to use a DCR (Kerr, Wood, Small, Palepu \& Tyndall, 2003). However, looking specifically at IDUs who had injected during the previous month, Wood et al. (2003) found that only 36.6\% were willing to use a DCR. DeBeck, et al. (2012) examined whether an initial willingness to use a DCR in Vancouver, prior to its opening, was associated with actual use of the facility subsequently. Of those who had claimed to be willing to use the facility before it opened, $72 \%$ reported attending the facility; moreover, 54\% of those who had reported being unwilling to use the DCR also reported attending. Willingness to attend predicted later attendance even after adjusting for other factors associated with willingness (e.g. age, gender, daily drug use, injecting in public locations). In the UK, Hunt, Lloyd, Kimber and Tompkins (2007) examined willingness to use a DCR in a sample of IDUs, most of whom were living in insecure housing and reported injecting in the previous week. The proportion willing to use a DCR if 
available (84\%) was similar to that reported by Kerr et al (2003). They also reported a greater willingness to use a DCR by users who routinely injected in public (although public injecting was also seen in those who lived in secure housing).

Whilst IDUs in other countries generally report in favour of DCRs there are a number of barriers to the opening of DCRs; these include political and legal issues and public resistance. Lissen et al.( 2001, as cited in Hedrich, 2004) noted that while DCRs in the Netherlands had a positive effect on public nuisance there was no effect on nuisance caused by drug dealing and general neighbourhood degeneration. Woods (2014) notes that at the time of set up, neighbours of DCRs in Europe showed more resistance to the facility than once it is established. The public's concerns often centre on the potential to attract drug dealers and drug users to the area. However, Hedrich (2004) argued that these concerns are largely unsubstantiated: as DCRs operate close to drug markets, some drug dealing has inevitably been reported near to them (Hedrich, 2004). The success of DCRs in reducing public nuisance is largely dependent on their opening hours, capacity and location (Hedrich et al, 2010); most have to deal with queues (Woods, 2014).

While there have been studies evaluating DCRs in Vancouver, Canada and Sydney, Australia and some studies investigating IDUs' attitudes and willingness to use DCRs in areas where DCRs were being considered or were about to be implemented, there has been limited research into the attitudes of IDUs towards DCRs in a country such as the UK where there are no DCRs at present and none planned in the foreseeable future. Furthermore, studies have mainly solicited views about DCRs from homeless IDUs or from those in insecure housing, or IDUs who are considered to be most at risk. The current study is novel as it examined the views of current and past IDUs, most whom were not homeless and lived in secure housing. The current study included IDUs who were not currently injecting as well as current injectors as a number of IDUs who have ceased injecting have been reported to relapse into injecting drug use, with longitudinal studies showing estimates of only $20-50 \%$ 
of injecting drug users (IDUs) who were drug free at follow up (Hser et al, 2001; Galai et al, 2003).

The aims of the present study were to survey the attitudes of IDUs in London (UK) to DCRs and to build on the findings of the IWG (2006). The study was not designed with any a priori view about promoting or arguing against DCRs or as a means to directly address the political argument surrounding DCR implementation in the UK. The study focused on three key aspects: IDUs’ willingness to use such facilities if they were available; how such willingness might be affected by specific DCR rules; and beliefs about how DCRs might impact on drug-injecting by oneself or others. For the third aspect, three specific ethical concerns about DCRs were considered: whether they would encourage non-injectors to inject; whether they would lead to the use of more drug or riskier practices; and whether they might encourage partners/friends to inject. These three ethical concerns have been identified by the independent working group (IWG, 2006) as important ethical issues deserving further investigation.

\section{Methods}

Participants

Participants were 90 intravenous drug users (IDUs) who attended a centre in London that provided methadone treatment, needle exchange, medical help and a range of advice services along with a drop-in service for dependent drug users. The centre was approached by the second author (DC) who was working as a volunteer. Participants were current or past injectors of at least one illicit drug and were all receiving methadone; they were approached in the methadone dispensary clinic during clinic hours, informed about the study and invited to take part; all of those who took part gave informed consent. Most participants $(\mathrm{N}=62$, or 69\%) were male with a mean age of 39.3 years (range 23-55 years); the mean age of the 28 
females was 37.3 years (range 21-58 years). Ninety-four per cent were white British, 2\% were black British, 3\% were other white race and data on ethnicity were missing for one participant. Eighty-four per cent were unemployed and 30.7\% were homeless. Only participants who did not exhibit overt signs of intoxication at the point of invitation were invited to take part. After participation they were provided with a voucher that could be exchanged for food in the cafeteria

\section{Materials and Procedure}

A 34-item questionnaire was designed for the study. The questions were informed by rules that commonly apply to DCR schemes in other countries and by previous research (e.g. van Beek \& Gilmore, 2000; Fry, 2002) and by concerns raised by the IWG (2006). The questionnaire assessed knowledge about DCRs (e.g. "What do you think a drug consumption room is?”), willingness to use a DCR, attitudes about rules commonly applied in DCRs, and beliefs about the impacts of DCRs on drug user behaviour. It also covered socio-demographic information and drug use history, including questions about injecting in public and private places, sharing of equipment, needle disposal practices and experiences of overdose. Eighteen questions required simple "yes” or "no" answers, and for thirteen of these questions - specifically relating to the various rules commonly implemented in DCRs - the participants were asked to provide reasons for their answers. Three other questions addressed important ethical concerns relating to the consequences of DCR provision, namely: (1) The possibility that DCRs would encourage non-injecting drug users to inject for the first time; (2) The possibility that DCRs would encourage IDUs to use higher drug doses, riskier preparations or riskier drug combinations than they would otherwise use; (3) The possibility that DCRs would encourage non-injecting partners/friends of IDUs to inject. Participants responded to each of these three questions by checking one of four boxes, labelled: "very high”, “quite high”, “quite low” or "very low”. 
Issues of data confidentiality, participant anonymity and the right to withdraw were strongly emphasised, and it was made clear that participation or not would have no bearing on their relationship with the centre in any way. Participation took approximately 30 minutes. Participants were assisted with filling in the questionnaire where there were literacy problems. The study was approved by the Research Ethics Committee of Kingston University Faculty of Arts and Social Sciences and also by the centre from which participants were recruited.

Results

Not all participants responded to every question, therefore the results are often expressed as percentages of the numbers who responded and of subsets who responded in particular ways.

Drug use characteristics of participants

At the time of participation, $68 \%(\mathrm{~N}=61)$ of the sample were still injecting drugs; $38 \%$ of these 61 reported injecting every day. Of the 32\% $(\mathrm{N}=29)$ of the sample who were not currently injecting, 6 had injected in the previous twelve months, and the remaining 23 had a history of intravenous drug use prior to that. The most commonly injected drug was heroin (identified by $82.2 \%$ of 74 respondents), followed by cocaine (20\% of 71 respondents), then amphetamine (8.9\% of 68 respondents). Seventy-seven participants provided information about the length of time that they had been injecting. The durations ranged from six months to 41 years $($ mean $=16.5$ years, $\mathrm{SD}=9.5$ years $)$. A substantial majority of those who responded had injected for more than 5 years (85.7\%); indeed, many had injected for over 20 years (41.6\%). Of the 66 participants who responded to a question asking about their normal methods of needle disposal, 73\% reported using only a needle exchange programme, with another 3\% using both needle exchange and litter bins. However, 20\% disposed of injection equipment in litter bins or tins only; the remaining 5\% used a sharps bin. Data from 66 participants indicated that $77 \%$ injected primarily in private locations whereas $23 \%$ injected 
in private or public places. However, despite the widespread preference to inject in a private place, $46 \%$ (of 68 respondents) reported having injected in a public place at least once in the previous month.

Knowledge of DCRs and willingness to use them

To determine whether the IDUs understood what was meant by a drug consumption room they were asked: "What do you think a drug consumption room is? (Also known as a safe injecting facility)”. Most participants responded to this question $(\mathrm{N}=87)$; of these, $85 \%$ claimed to be familiar with the idea of DCRs. Of the participants who knew about DCRs, 81\% identified DCRs as safe environments in which to inject drugs, $12 \%$ simply reported them as places to inject. DCRs were described as "not a good idea” by $4 \%$ and as "a drug den by 3\%. After clarifying what participants understood by a DCR, 77 out of 87 (i.e. 89\%) indicated that they would be willing to use a DCR. Of the 61 participants who were still injecting at the time of data collection 95\% indicated that they would be willing to use a DCR, whereas $73 \%$ of the 26 participants who were no longer injecting declared a willingness to use a DCR $\left(\chi^{2}(1)=8.68, \mathrm{~N}=87, \mathrm{p}=.003\right)$. There was an overwhelming preference expressed for injecting in a DCR over a public place: 83 of 88 participants who responded (94\%). However, only $26.7 \%$ (24 of the 88 ) reported that they would prefer to inject in a DCR rather than in a private place.

Attitudes towards rules applied by DCRs

Substantial majorities of participants stated that they would be willing to use DCRs with the most common rules that currently operate in most DCRs: Compulsory handwashing $(92.1 \%$ of 89 respondents); No help with injecting (81.8\% of 90 respondents); Compulsory supervision (76.7\% of 90 respondents). A large majority, 84.3\% (out of 89 respondents), 
were also willing to use a DCR if drug sharing was not permitted, albeit that 5 of 45 respondents (11.1\%) who gave a reason said that they would split/share drugs before entering the facility. Participants were also asked if users of the DCR should not be allowed to inject in the neck or groin: of the 85 participants who responded to the question, $71.8 \%$ (61) disagreed with the rule. Of the 61 participants who disagreed, $70.5 \%$ gave as their reason the fact that the neck and groin were the only places left to inject for some IDUs.

Finally, participants were asked if certain categories of IDUs should be not allowed to use a DCR and whether or not health checks should be compulsory for users. Table 1 shows that there was support for access by novice users and non-residents, as only a minority agreed these groups should not be allowed access to the DCR, but the sample was split over allowing access to people under the age of 18 years old or to pregnant women. Participants ( $\mathrm{N}=88$ respondents) were also quite evenly divided over the issue of compulsory health checks for access to facilities: 46 (52.3\%) agreed and 42 (46.7\%) disagreed.

\section{----- TABLE 1 APPROXIMATELY HERE ----}

Views on ethical concerns expressed about the impacts of DCRs For each of the three ethical concerns, analyses were conducted to compare the views of the 61 current IDUs and the 29 participants who were not currently injecting. There were no statistically significant differences between the two groups on any of the three issues, hence their results will be considered together.

1. Will DCRs encourage injecting by non-injectors? Participants were asked if they thought that DCRs would attract non-injectors to inject for the first time, taking into account that DCRs would not allow assistance with injecting. Only about one-quarter thought it probable (10\% 
rated the possibility as “very high”, 15.6\% rated it “quite high”); whereas nearly three-quarters thought it unlikely (35.6\% rated the possibility “quite low” and 38.9\% “very low”).

2. Will DCRs encourage riskier drug use by injectors? The issue of whether DCRs might encourage users to take greater quantities of drugs or to use more risky preparations or combinations was assessed. The split in views was similar to the previous question: the likelihood was rated as “very high” by $10 \%$, “quite high” by $13.3 \%$; “quite low” by $42.2 \%$ and “very low” by 34.4\%.

3. Will DCRs encourage non-injecting partners/friends to inject? Participants were asked whether non-injecting partners/friends of IDUs might be tempted to inject in a DCR if available. Here, the sample was more evenly divided between those who agreed and those who disagreed: $14.4 \%$ rated the likelihood as “very high”, $28.9 \%$ as “quite high”, $32.2 \%$ as "quite low" and $24.4 \%$ as "very low.

\section{Discussion}

The study found that the majority of IDUs knew what a DCR was and indicated a willingness to use a DCR if one was established. The high level of willingness to use a DCR is consistent with the findings of Hunt et al. (2007), also with a UK sample, as well as with findings from other countries (van Beek \& Gilmore, 2000; Green et al., 2004; Fry, 2002; Kerr et al., 2003; DeBeck et al., 2012). A significantly higher proportion of current IDUs expressed willingness to use DCRs than those who were not currently injecting, although the question did not ask them to reflect on their view as if they were still injecting. The majority of participants also stated that they were willing to use DCRs if rules were implemented, including no assistance with injecting. The majority of IDUs also showed willingness to use a DCR if handwashing was compulsory and if supervision was compulsory. Very few IDUs expressed concerns about prohibiting drug sharing/splitting at the DCR, although a small proportion admitted that 
they were likely to split their drugs before entering the DCR. Kerr et al (2003) found that over 60\% were willing to use DCRs even if drug sharing and assisted injection were prohibited; similarly, Kral et al. (2010) reported that $67 \%$ of IDUs would accept a rule that drug sharing is not allowed . Fry (2002) found that IDUs were willing to use a DCR if they were prevented from assisting others to inject, as long as the DCR was close to where the user purchased and used drugs. Fry (2002) also found that just over half were still willing to use a DCR if they were prevented from splitting drugs on the premises, a lower proportion than in the current study. Overall, the current study, of past and present IDUs in the UK, indicates similar levels of willingness to use DCRs under similar rules as in other countries. This lends support to the view that DCRs might yield similar benefits in the UK.

Where IDUs showed disagreement with probable DCR rules was in relation to the prohibition of injection in the neck or groin. Most participants (72\%) disagreed with this rule, the main reason being that for some users these were the only places left to inject. This result may reflect the fact that whilst some users are forced to inject in the neck and groin as a result of long-term injecting, it has become increasingly normal in some English cities (Rhodes et al., 2006). Maliphant and Scott (2005) reported that 51\% of their UK sample of IDUs was injecting in the groin because no other convenient sites were considered to be accessible and the likelihood of heroin users injecting into sites other than the arm increases with the duration of injecting (Karimi et al., 2014). Therefore this is an issue which will need addressing by all DCRs as many target long term and problematic injectors and prohibit injecting into the neck and groin.

IDUs in the current study were asked whether they agreed that certain people should not be allowed to use a DCR. Around three-quarters disagreed with a rule that novice injectors and non-residents should not be allowed to use a DCR. The proportion that felt nonresidents should not be able to use a DCR, around one quarter, is lower than among a sample 
of IDUs in San Francisco, USA, where 34\% agreed that users of a DCR should live in the neighbourhood (Kral et al., 2010). The difference between these two samples, London and San Francisco, may be due to the higher number of homeless people in the San Francisco sample, where the majority was homeless. In contrast nearly half in the current study agreed that pregnant women should not be permitted to use a DCR and just over half agreed that those under 18 years should not be permitted to use a DCR. These attitudes against pregnant women or non-residents being allowed to access the facility are not in line with the findings from a stakeholder survey in Canada (Fisher \& Allard, 2007). That study showed the majority of stakeholders thought that pregnant women should be permitted to use a DCR, and that there should be as few barriers as possible to using a DCR. Zadjow (2006) noted that, at the time of their study, Sydney was the only DCR which did not allow access for pregnant women. There is clearly some difference of opinion as to who should be allowed to use a DCR. The differences are likely to reflect location-specific factors concerning the composition of the local drug using community, levels of homelessness and broader national/local policies for managing the treatment of drug misuse. The findings indicate a need for dialog with the likely users of new facilities when developing regulations for access to the DCR; such regulations are likely to vary across locations.

Although the majority of participants cited a private place as the usual place of injecting, $40 \%$ had nevertheless injected in a public place in the last month. Clearly, public injecting is not limited to the homeless and is not an exclusive choice by a subset of users, it is common amongst IDUs. It is important therefore that most participants in the current study reported a preference for using a DCR over a public place to inject. Similar outcomes were found in Canada and Australia (van Beek \& Gilmore, 2000; Green et al., 2004). A report from a UK sample indicated that public injecting was associated with insecure housing (Hunt et al., 2007), but in the current study only thirty percent of the sample was homeless and the 
majority lived in secure housing. Together, these results suggest that the majority of IDUs do not like to inject in public places but do so out of necessity due to a sense of urgency to inject and no other suitable place near to the drug market. Therefore DCRs are likely to reduce public injecting and to decrease the nuisance and litter associated with public injecting.

The majority of participants felt that the probability of DCRs encouraging injecting by non-injectors was low. This may be because first-time injectors typically require assistance, and this is not permitted by most DCRs. Crofts et al. (1996) reported that only $12 \%$ of IDUs injected themselves the first time; Kermode et al. (2007) reported that $94.5 \%$ of IDUs in their study were injected by someone else first time. They also found that the majority had helped someone else inject for the first time. Therefore the concern that DCRs would attract noninjecting users to inject is not supported by the views of IDUs in this study and is consistent with previous research. A second ethical issue addressed was whether DCRs would encourage riskier drug use, such as taking greater quantities of drugs or riskier combinations or preparations. The likelihood was rated low by over three-quarters of participants, consistent with other reports that DCRs do not encourage riskier patterns of use (Hedrich, 2004) and might in fact lead to less risky practices: the medically supervised injecting centre (MSIC, 2003) in Sydney found that almost half of the users of the centre reported less risky injecting practices since they began using the centre.

The final ethical issue examined was whether DCRs would encourage non-injecting friends or partners of IDUs to inject. Participants were divided on this issue, with just over half rating the likelihood as low. If a person is thinking of injecting for the first time, then a medically supervised site may offer some sense of security if something goes wrong. However as most DCRs have rules which do not allow assistance with injecting and many first time injectors require or have assistance then this situation would be unlikely. Furthermore the MSIC in Sydney has a policy of engaging with the non-injecting drug users 
who try to use the centre, assessing them and providing referral to services to discourage the transition to injecting (KPMG, 2010). Any DCR should be able to implement an analogous policy. Overall, these findings relating to the ethical concerns raised by the IWG suggest that the concerns are not supported by the evidence.

There are a number of inevitable limitations with the current study. For example, it was reliant on self-report and there were no objective records available to verify details such as length of time injecting or if the participants were drug free at the point of data collection. However, other studies in settings where there is no client-health care provider relationship have found that self-reported drug use typically yields valid measures (Langendam et al., 1999). Some caution is needed in comparing across studies. The composition of the sample in the present study was different from that in other studies which often include higher proportions of public injectors, at-risk IDUs and homeless people. The present sample also included people who were no longer injecting, and although they expressed less support for DCR use than current injectors, the findings for the sample as a whole were largely consistent with those reported elsewhere. Despite the sample differences, willingness to use DCRs was high and willingness to use if rules were in place was higher than in some other studies. The duration of injecting by IDUs was also consistent with other studies looking at long term IDUs. There were no significant differences between the views of those currently injecting compared to those who were not currently injecting on their attitudes regarding the three ethical concerns about DCRs. The views of those no longer injecting are important since, as experienced past injectors, they can provide a particular perspective on how DCRs might have impacted on their own trajectory towards abstinence; moreover, longitudinal studies suggest that significant numbers of abstinent drug users will relapse to injecting drug use (Galai et al, 2003; Hser et al, 2001) including those receiving methadone treatment (Gossop et al, 2003: Termorshuizen et al, 2005). A more recent consideration for those implementing 
new DCRs is the increase in the smoking of drugs (Hedrich et al., 2010). Although some established DCRs have booths for the smoking of drugs others do not and whether newly established DCRs would allow the smoking of crack or heroin or whether separate DCRs would operate for these groups of drug users, are all issues that would need to be considered as DCRs respond to changes in drug use.

The findings have shed important light on the likely uptake of DCRs, and the implications are positive. Evaluations of DCRs in other countries have shown them to be costeffective and to provide a range of benefits to IDUs and the communities in which they operate. Evaluation of such facilities prior to opening and also during and after a trial period would be necessary, in consultation with the appropriate stakeholders: users, service providers and local residents and businesses.

\section{Acknowledgment}

The authors would like to thank all the participants who took part in this study.

\section{Funding}

There was no external funding supporting this research.

\section{Conflict of interest}

The authors report no conflicts of interest.

References

Broe, T. (2016). Making the case for drug consumption rooms. The Pharmaceutical Journal, 296, No 7889, online. DOI: 10.1211/PJ.2016.20201185.

Crofts, N., Louie, R., Rosenthal, D., \& Jolley, D. (1996). The first hit: circumstances surrounding initiation into injecting. Addiction, 91, 1187-1196. doi: 10.1046/j.13600443.1996.918118710.x 
Cusick, L. \& Kimber, J. (2007). Public perceptions of public drug use in four UK urban sites. International Journal of Drug Policy, 18, 10-17. doi:10.1016/j.drugpo.2006.12.017

DeBeck, K., Kerr, T., Lai, C., Buxton, J., Montaner, J., \& Wood, E. (2012). The validity of reporting willingness to use a supervised injecting facility on subsequent program use among people who use injection drugs. American Journal of Drug and Alcohol Abuse, 38, 55-62. doi:10.3109/00952990.2011.600389

Dolan, K., Kimber,J., Fry, C., Fitzgerald,J., McDonald, D., \& Trautmann, F. (2000). Drug consumption facilities in Europe and the establishment of supervised injecting centres in Australia. Drug and Alcohol Review, 19, 337-346. http://dx.doi.org/10.1080/713659379

EMCDDA (2012). Annual Report The State of the Drugs Problem in Europe. Lisbon: European Monitoring Centre for Drugs and Drug and Addiction.

Fisher, B., \& Allard, C.A. (2007). Feasibility Study on 'Supervised Drug Consumption' Options in the City of Victoria. Centre for Addictions Research of British Columbia (CARBC), University of Victoria.

Fry, C.L. (2002). Injecting drug user attitudes towards rules for supervised injecting rooms: implications for uptake. International Journal of Drug Policy, 13, 471-476. http://dx.doi.org/10.1016/S0955-3959(02)00076-2

Galai, N., SafaeianM., Vlahov,D., Bolotin, A. \& Celentano, D.D. (2003). Longitudinal patterns of drug injection behavior in the ALIVE study cohort, 1988-2000: Description and Determinants. American Journal of Epidemiology, 158, 695-704. doi: 10.1093/aje/kwg209

Gossop, M., Marsden, J., Stewart, D \& Kidd, T. (2003). The National Treatment Outcome Research Study (NTORS): 4-5 year follow up results. Addiction, 98, 291-303. doi: 10.1046/j.1360-0443.2003.00296.x 
Green, T.C., Hankins, C.A., Palmer, D., Boivin, J-F., \& Platt, R. (2004). My Place, Your Place, or a Safer Place. The Intention Among Montréal Injecting Drug Users to Use Supervised Injecting Facilities. Canadian Journal of Public Health, 95, 110-114. Retrieved from http://www.jstor.org/stable/41994109

Hedrich, D. ( 2004). European Report on Drug Consumption Rooms. Lisbon: European Monitoring Centre for Drugs and Drug Addiction.

Hedrich, D., Kerr, T., \& Dubois-Arber, F. (2010). Drug consumption facilities in Europe and beyond. In Rhodes, T \& Hedrich, D (Eds.), 306-331. Harm reduction: evidence, impact and challenges EMCDDA Monographs 10 (pp. 306-331). Lisbon: EMCDDA.

Hser, Y. I., Hoffman, V., Grella, C. E., \& Anglin, M. D. (2001). A 33-year follow-up of narcotics addicts. Archives of general psychiatry, 58(5), 503-508. doi:10.1001/archpsyc.58.5.503

Hunt, N., Lloyd, C., Kimber, J., \& Tompkins, C. (2007).Public injecting and willingness to use a drug consumption room among needle exchange programme attendees in the UK. International Journal of Drug Policy, 18, 62-65. doi:10.1016/j.drugpo.2006.11.018

Hunt, N. (2006). Independent Working Group on Drug Consumption Rooms Paper A. Indicators of the need for drug consumption rooms in the UK. York: Joseph Rowntree Foundation.

IWG. (2006). The report of the Independent Working Group on drug consumption rooms. York: Joseph Rowntree Foundation.

Karimi, M., Ghaheri, H., Assari, S., Ahmadi, K., Moghani, L., Lankarani, R.M., Nenejiha, H., Rafiey, H., Tavakoli, M., \& Jafari, F. (2014). Drug injection to sites other than arm: a study of Iranian heroin injectors. Frontiers in Psychiatry, 5, 1-5. doi: 10.3389/fpsyt.2014.00023 
Kral, A. H., Wenger, L., Carpenter, L., Wood, E., Kerr, T., \& Bourgois, P. (2010). Acceptability of a Safer Injection Facility among Injection Drug Users in San Francisco. Drug and Alcohol Dependence, 110, 160-163. doi:10.1016/j.drugalcdep.2010.02.009

Kermode, M., Longleng, V., Singh, B.C., Hocking,J., Langkham, B., \& Crofts, N. (2007). My first time: initiation into injecting drug use in Manipur and Nagaland, north-east India. Harm Reduction Journal, 4, 19. doi:10.1186/1477-7517-4-19

Kerr, T., Wood, E., Small, D., Palepu, A., \& Tyndall, M.W. (2003). Potential use of safer injecting facilities among injection drug users in Vancouver's Downtown Eastside. Canadian Medical Association Journal, 169, 759-763.

Kerr,T., Tyndall, M., Li, K., Montaner, J., \& Wood, E. (2005). Safer injection facility use and syringe sharing in injection drug users. Lancet, 366, 316-318. doi:10.1016/S0140 $-6736(05) 66475-6$

Kerr, T., Stoltz, J., Tyndall, M., Li, K., Zhang, R., Montaner, J., \& Wood, E.(2006). Impact of a medically supervised safer injection facility on community drug use patterns: a before and after study. British Medical Journal, 332, 220-2. doi:10.1136/bmj.332.7535.220

KPMG. (2010). Further evaluation of the Medically Supervised Injecting Centre during its extended Trial Period (2007-2011). Prepared for NSW Health by KPMG, September 2010.

Latkin, C., Mandell, W., Vlahov, D., Oziemkowska, M., Knowlton, A., \& Celentano, D. (1994). My place, your place, and no place: behavior settings as a risk factor for HIVrelated injection practices of drug users in Baltimore, Maryland. American Journal of Community Psychology, 22, 415-30. doi: 10.1007/BF02506873 
Lloyd, C., \& Hunt, N. (2007). Drug consumption rooms: An overdue extension to harm reduction policy in the UK? International Journal of Drug Policy, 18, 5-9. doi: 10.1016/j.drugpo.2006.12.010

Maliphant , J., \& Scott, J. (2005).Use of the femoral vein ('groin injecting') by a sample of needle exchange clients in Bristol, UK. Harm Reduction Journal, 2, 6. doi:10.1186/1477-7517-2-6

McLellan, A.T., Lewis, D.C., O’Brien, C.P. Kleber, H.D. (2000). Drug dependence, a chronic medical illness: implications for treatment, insurance and outcomes evaluation. JAMA, 284, 1689-1695. doi:10.1001/jama.284.13.1689

MSIC Evaluation Committee. (2003). Final report on the evaluation of the Sydney Medically Supervised Injecting Centre. Sydney: authors.

Petrar, S., Kerr, T., Tyndall, M.W., Zhang, R., Montaner, J.S.G., \& Wood, E. (2007). Injection drug users' perceptions regarding use of a medically supervised safer injecting facility. Addictive Behaviours, 32, 1088-1093. doi:10.1016/j.addbeh.2006.07.013

Pinkerton, S.D.(2010). Is Vancouver Canada’s supervised injection facility cost-saving? Addiction, 105, 1429-1436. doi:10.1111/j.1360-0443.2010.02977.x

Potier, C, Laprevote, V, Dubois-Arber, Cottencin, O, Rolland, B. (2014). Supervised injection services: What has been demonstrated? A systematic review. Drug and Alcohol Dependence, 45, 48-68. doi:10.1016/j.drugalcdep.2014.10.012

Rhodes, T., Stoneman, A., Hopea,V., Hunt, N., Martin, A., \& Judd, A. (2006). Groin injecting in the context of crack cocaine and homelessness: From 'risk boundary' to ‘acceptable risk’? International Journal of Drug Policy, 17, 164-170. doi:10.1016/j.drugpo.2006.02.011

Termorshuizen, F., Krol, A., Prins, M., Geskus, R. van den Brink, W. \& van Ameijden, E.J.C. (2005). Prediction of relapse to frequent heroin use and the role of methadone 
prescription: An analysis of the Amsterdam Cohort Study among drug users. Drug and Alcohol Dependence, 79, 231-240. doi:10.1016/j.drugalcdep.2005.01.013

Van Beek, I., \& Gilmour, S. (2000). Preference to have used a medically supervised injecting centre among injecting drug users in Kings Cross, Sydney. Australian and New Zealand Journal of Public Health, 24, 540-542.doi: 10.1111/j.1467842X.2000.tb00507.x

Wood, E., Kerr, T., Spittal, P.M., Li, K., Small, W., Tyndall, M.W., Hogg, R.S., O’Shaughnessy, M.V., \& Schechter, M.T. (2003). The potential public health and community impacts of safer injecting facilities: Evidence from a cohort of injection drug users. Journal of acquired immune deficiency syndromes, 32, 2-8. doi.org/10.1097/00126334-200301010-00002

Wood, E., Kerr, T., Small, W., Li, K. , Marsh, D.C., Montaner, J.S.G., \& Tyndall, MW. (2004). Changes in public order after the opening of a medically supervised safer injecting facility for illicit injection drug users. Canadian Medical Association Journal, 171, 731-4. doi: 10.1503/cmaj.1040774

Wood, E., Tyndall, M.W., Li, K., Lloyd-Smith, E., Small, W., Montaner, J.S.G., \& Kerr, T. (2005). Do Supervised Injecting Facilities Attract Higher-Risk Injection Drug Users? American Journal of Preventive Medicine, 29, 126-130. doi:10.1016/j.amepre.2005.04.011

Woods, S. (2014). Organisational overview of dug consumption rooms in Europe. European Harm Reduction Network .Regenboog Groep:Amsterdam (www.eurohrn.eu/index.php/drug-consumption-rooms).

Zadjow, G. (2006). The narrative of evaluations: medically supervised injecting centers. Contemporary drug problems, 33, 399-426. 